\title{
LOTES Y CONJUNTOS RESIDENCIALES COMO POTENCIALES GENERADORES DE IMPACTOS DE VECINDAD
}

\author{
José Augusto de Lollo \\ Facultad de Ingeniería de Ilha Solteira- UNESP \\ Sérgio Antonio Röhm \\ Centro de Ciencias Exactas y de Tecnología- UFSCar
}

\section{RESUMEN}

La proposición o instalación de parcelamientos y conjuntos residenciales en Brasil, es objeto de instrumentos de gestión tanto del punto de vista legal como técnico desde hace tiempo. Tales instrumentos prevén restricciones, limitaciones, y reglamentaciones, cuyo interés ha sido proteger los patrimonios natural y cultural y verificar la adecuación de la propuesta a preceptos urbanísticos. En la mayoría de los casos tales leyes son aplicadas solamente a las actuaciones de gran porte. Con la promulgación de Ley 10.257/2001, impactos ambientales, antes desconsiderados pasan a tener importancia, obligando los municipios brasileños a elaborar o revisar sus planes directores hasta Octubre de 2006. Nuevas categorías de impacto deben ser consideradas y las técnicas de identificación y evaluación de tales impactos en áreas urbanas deben ser revisadas, para dara cumplimento a las exigencias actuales y futuras de la legislación municipal. Propuestas de conjuntos habitacionales de menor porte, antes dispensadas de presentar tales estudios, deben ahora ser cuidadosamente evaluadas. Sólo la consideración de todos los aspectos posibles de modificación permitirá evaluaciones apropiadas de tales propuestas de ocupación, y la consecuente reducción de impactos para la población.

Palabras clave: parcelamientos, impacto ambiental, impacto de vecindad, planeamiento urbanístico, habitación popular.

\begin{abstract}
The proposition and installation of divisions into lots in Brazil is considered by several administration instruments as much of legal or technician points of view from long date. Such instruments foresee restrictions, limitations, and regulations whose interest has been to protect natural and cultural patrimonies and to verify the adaptation of the proposal to town planning precepts. Law 10.257/2001 promulgation created a new situation in which impacts no before foreseen need to be taken in account and Brazilian counties need pro-
\end{abstract}


pose or adapt its director plans until October 2006. Thus, new impact categories should be considered while identification and evaluation techniques for such impacts need to be revised, in order to treat new municipal legal precepts. New smaller proposed districts, whose impacts aren't discussed in the past, now need careful evaluation. Considering all the striped aspects will allow appropriate evaluations of such occupation proposals, reducing impacts for population.

Key-words: divisions into lots, environmental impacts, neighborhood impacts, urban planning, popular house.

\section{Introducción}

Se puede decir que la preocupación en disciplinar la parcelación urbana existe en Brasil desde que las ciudades brasileñas se consolidaron como agrupamientos urbanos del punto de vista de su infraestructura básica, proceso que se inició, todavía, en el tiempo de la colonización.

En especial, lo que dice respecto a la legislación federal el asunto presenta un histórico largo de leyes, decretos y códigos que vienen intentando disciplinar tal tipo de ocupación en pro de la mejoría de las condiciones urbanísticas.

El Consejo Nacional del Medio Ambiente, a partir de su creación en 1986, y considerando las cuestiones ambientales relacionadas a los parcelamientos, pasó también a considerar, en los objetivos de sus resoluciones, que tratan de las cuestiones ambientales, en áreas urbanas, los parcelamientos como propuesta de ocupación con posibilidad de regulación. Al mismo tiempo, Estados y Municipios brasileños han buscado alternativas para disciplinar la cuestión, sea en la forma de leyes y decretos municipales o estatales, sea en la forma de proposición de reglamentos y procesos de análisis por secretarías de estado y municipio.

Incluso con todo ese cuidado e interés del poder público, algunos tipos de impactos consecuentes de la implantación de conjuntos residenciales todavía no son debidamente tratados, sea por ineficacia de los instrumentos legales, sea por falta de preparación del sector técnico de entidades públicas y empresas privadas.

Con la promulgación de la Ley 10.257/2001 (llamada «Estatuto de la Ciudad») una categoría de impactos ya discutida por el medio técnico pasó a ser considerada como instrumento legal de gestión urbana, el Estudio del Impacto de Vecindad.

La Ley 10.257/2001 estableció los principios generales para tal tipo de análisis al proponer que los municipios brasileños definan, en legislación municipal, los tipos de emprendimientos de los cuales será exigida y realización de tales estudios. La Ley define cuales los factores ambientales a ser evaluados cuando de la realización del Estudio Previo de Impacto de Vecindad, y somete el licenciamiento de tales actividades a su realización (Soares Filho, 2001).

La naturaleza de los factores citados en la Ley representa la necesidad de expandir los estudios ambientales relacionados a propuestas de implantación de conjuntos residenciales, considerando atributos no citados tradicionalmente. Tales avances significan la necesidad de ampliación o adecuación de las formas de levantamiento y análisis de datos, para atender a las nuevas necesidades.

Se pretende discutir la cuestión de la evaluación de impactos ambientales en áreas urbanas debidos a la implantación de conjuntos residenciales, presentando una revisión de la legislación existente, considerando la legislación «tradicional» y la nueva realidad creada con la Ley 10.257/2001. 
También se discute la forma de conducción de los trabajos de evaluación de impactos para tales propuestas de ocupación y uso del suelo, en lo que dice respecto a los atributos a ser considerados; la forma de levantamiento de los mismos; y las análisis y interpretaciones juzgadas más apropiadas por los autores.

\section{Legislación relacionada}

\subsection{Legislación Federal Brasileña}

A pesar de las primeras leyes de protección a la naturaleza existieren desde los tiempos de la colonización en Brasil (como las Ordenaciones Manuelinas del Rey Don Manuel, que prohibía la caza de ciertos animales, las Ordenaciones Filipinas del Rey Felipe II que prohibía cualquier persona de tirar material que pudiera matar a los peces), la legislación que rige del ordenamiento urbano surgió solamente en el siglo XX (Boccasius-Siqueira, 2002).

La legislación federal que rige el asunto puede ser dividida en dos grupos. En el primero se encuentran los instrumentos legales que tratan las restricciones o recomendaciones existentes cuanto a la localización de conjuntos habitacionales, y en el segundo grupo aquellos que tratan de los medios y procedimientos para que tales propuestas se den.

\subsubsection{Restricciones a la Implantación}

La referencia más antigua de restricción de uso del suelo urbano por construcción civil puede ser encontrada en el Código de Aguas, en el Decreto 24.643/34 (Brasil, 1934), y se refiere a la prohibición, a los propietarios de terrenos en las márgenes de los ríos y riachuelos, de construir cualquier edificación que pueda impedir o dificultar el libre curso de las aguas.

El Decreto- ley 25/37 (Brasil, 1937), con la intención de proteger el Patrimonio Histórico y Artístico Nacional, prohíbe la construcción, en la vecindad de la cosa amparada, de cualquier cosa que impida o reduzca la visibilidad del elemento protegido.

La Ley 4.771/65, que estableció el Código Forestal (Brasil, 1965a), establece los criterios para la definición de áreas de protección permanente, las cuales no deben ser ocupadas por actividades humanas que signifiquen la supresión de especies vegetales. Y también del año de 1965 la Ley 4.778 (Brasil, 1965b) que define la consulta a las autoridades forestales para licenciamiento de parcelamientos en áreas forestadas.

Cuanto a la forma de ocupación del área por un parcelamiento urbano, el Decreto- ley 271/67 (Brasil, 1967) permite que los municipios subordinen la implantación de los parcelamientos a criterios de orientación y utilización de las áreas, o que impidan su instalación si el numero de parcelas cause gran impacto en la infraestructura urbana.

\subsubsection{Medio y Procedimientos para la implantación}

Con relación a la forma como las ocupaciones por parcelamientos y conjuntos habitacionales, se debe dar, la referencia más antigua encontrada es la Ley 4.591/64, que establece que, en el uso de su unidad del condominio, el propietario no puede causar daño o incomodo a los demás residentes (Brasil, 1964).

En 1979 fue promulgada la Ley 6.766 (Brasil, 1979) que dispone sobre la parcelación del suelo urbano y define los criterios fundamentales de la ocupación urbana. La ley delega a la legislación municipal el poder de definir las áreas disponibles para la ocupación, pero 
no permite la parcelación del suelo en áreas inundadas, antiguos terraplén de materiales nocivos, terrenos con declinación más grande que 30\%, terrenos con condiciones geológicas inadecuadas, y áreas de preservación. La ley define criterios básicos (como dimensiones de parcelas), áreas no edificables, criterios de adaptación de la propuesta de ocupación a la infraestructura urbana existente.

Todavía lo que se dice respecto a las limitaciones a la ocupación, la Ley 6.766 remite a los Estados la decisión en relación de la ocupación de áreas limítrofe de municipios, de interés especial, y parcelamientos con áreas superiores a $1.000 .000 \mathrm{~m}^{2}$. El Decreto 86.176/81 (Brasil, 1981a) dispone sobre la creación de áreas de interés turístico y reglamenta la protección de tales áreas de los proyectos de ocupación habitacional. La Ley 6.938 (Brasil, 1981b) formula la Política Nacional del Medio Ambiente y remite al CONAMA (Consejo Nacional del Medio Ambiente) competencia para definir criterios y normas para licenciamiento de actividades potencialmente contaminante.

El Decreto 99.274/90 (Brasil, 1990) establece la exigencia de elaboración de Estudios de Impacto Ambiental para propuestas de ocupación que sean potencialmente contaminantes para la evaluación por órganos del Sistema Nacional del Medio Ambiente. El Decreto 1.922/96 (Brasil, 1996) establece los criterios para la definición de Reservas Particulares del Patrimonio Natural, las cuales no pueden ser ocupadas por actividades de ocupación sin estudios específicos para tal finalidad.

En 1997 la Ley 9.433 (Brasil, 1997), al instituir la Política Nacional de Recursos Hidráulicos, define como infracción la derivación o la utilización de recursos hidráulicos (superficiales y subterráneos) sin otorga de derecho de uso, bien establece criterios para la implantación de emprendimiento relacionado al uso de recursos hidráulicos. La Ley 9.785/99 (Brasil, 1999) establece criterios para la implantación de infraestructura básica de las parcelaciones ubicadas en Zonas Habitacionales de Interés Social, y sugiere que esta parcelación sea definida en el Plan Director del Municipio.

\subsection{Resoluciones del CONAMA}

También en el caso de las resoluciones del CONAMA la legislación puede ser compartida en tres categorías: resoluciones que tratan de restricciones para la implantación de parcelamientos y conjuntos residenciales: Legislaciones que definen los estudios a ser realizados visando la implantación de tales propuestas: y aquellas que tratan de las formas de reparación de daños ambientales a causa por la ocupación.

\subsubsection{Restricciones a la implantación}

La Resolución 011/87 (CONAMA, 1987b) define una serie de áreas, estaciones y reservas que son consideradas Unidades de Conservación, por lo tanto protegidas de ocupación. La prohibición de actividad que pueda poner en riesgo la conservación de los ecosistemas, protección del biótopo y la armonía del paisaje de las áreas de Relevante Interés Ecológico fue definida por la Resolución 12/89 (CONAMA, 1989).

La Resolución 02/02 (CONAMA, 2002a) amplia los criterios de definición de Áreas de Preservación Permanente además de los cursos de agua y estanques naturales, para el alrededor de los estanques artificiales, creando así limitaciones a la ocupación en esas áreas. Con relación a límites de Áreas de Preservación Permanente, la Resolución 302/02 (CONAMA, 2002b) amplía los tipos de terrenos protegidos, pasando a incluir situaciones como líneas de cumbre, escarpas de tableros e altiplanicies, restingas, áreas de dunas y manglares, y locales de interés para la preservación de especies. 
Con la intención de preservar especies vegetales raras o amenazadas, la Resolución 339/03 (CONAMA, 2003a) protege el alrededor de los Jardines Botánicos, que deben contar con áreas anexas preservadas, por lo tanto exentas de ocupación.

\subsubsection{Estudios a Ser Realizados}

Con relación a los estudios ambientales, el gran momento en la legislación brasileña es la Resolución 01/86 (CONAMA, 1986) que estableció los emprendimientos a la obligación de la elaboración de Estudios de Impacto Ambiental, incluyendo ahí los proyectos urbanísticos con áreas superiores a 100ha o en áreas consideradas de relevante interés ambiental.

La Resolución 05/88 (CONAMA, 1988) establece que obras de saneamiento en las cuales sea posible identificar cambios ambientales significativos están sujetas a licenciamiento ambiental, incluyéndose ahí Sistemas de Abastecimiento de Agua, de Alcantarillas Sanitarias, Sistemas de Drenaje, y Sistemas de Limpieza Urbana.

Con relación a la permisión de actividades potencialmente agresoras al medio ambiente, la Resolución 13/90 (CONAMA, 1990) define cualquier actividad que pueda afectar el biótopo en una área ubicada a una circunferencia de $10 \mathrm{~km}$ de las Unidades de Conservación Ambiental está sujeto a la autorización del órgano ambiental competente.

La Resolución 04/93 (CONAMA, 1993) obliga a la aprobación de los órganos ambientales las actividades, obras, planes y proyectos a ser instalados en restingas. Con relación a las actividades que utilicen de recursos naturales o que sean potencialmente contaminadoras, la Resolución 237/97 incluye las actividades de parcelación del suelo como dependientes de la realización de Estudios de Impacto Ambiental.

Con respecto a producción de residuos de construcción, la Resolución 307/02 (CONAMA, 2002c) establece condiciones para disposición de los residuos. La Resolución 341/03 (CONAMA, 2003b) establece criterios y estudios para ocupación de áreas de dunas, obligando la realización de Estudios de Impacto Ambiental.

Con el objetivo de proteger el patrimonio espeleológico, la Resolución 347/04 (CONAMA, 2004) define criterios para licenciamiento ambiental de actividades que se encuentren en el área de influencia de grutas y cavernas.

\subsubsection{Formas de Reparación de Daños}

La Resolución 02/96 (CONAMA, 1996) define que las actuaciones consideradas (con fundamento en el Estudio de Impacto Ambiental) de relevante impacto ambiental por el órgano ambiental competente deberán a título de forma de reparación de los daños ambientales, emprender la instalación de una Unidad de Conservación, donde el importe de recursos invertidos en la unidad proporcional al daño ambiental, no puede ser inferior a $0,5 \%$ de los costos totales para la implantación del emprendimiento.

Efectivamente, tal propuesta de reparación de daño ya había sido considerada por la Resolución 10/87 (CONAMA, 1987a), sin embargo esa definía los emprendimientos como «obras de gran porte», limitando en mucho la aplicación de la resolución, a lo contrario de la Resolución 02/96 que adopta la descripción «emprendimiento de relevante impacto ambiental», sin considerar su porte.

\subsection{Legislación de Estados y Municipios Brasileños}

Los estados brasileños, con el fin de definir, en su esfera de acción, criterios de defensa y conservación de la calidad ambiental, tiene, desde la década de 80, creado legislación para tratar del asunto. 
Tal legislación, que en inicio se basaba esencialmente en leyes y decretos, evolucionó para su consolidación en la forma de los Planes Directores de los Municipios, que intentan contemplar los factores de interés para la defensa del medio ambiente municipal.

\subsubsection{Características de la Legislación de los Estados}

La legislación estatal, en la gran mayoría de los casos, tiene como principio definir criterios generales y competencias, preservando el poder de los municipios de legislar en defensa del interés específico de cada uno de ellos.

Por tratarse de materia vasta, dado el número de Estados que la República Brasileña posee, y las particularidades que el tema puede presentar, se optó por apenas describir sus características comunes y presentar algunos ejemplos.

La característica común más evidente en la legislación de los estados brasileños que trata de la parcelación y ocupación del suelo urbano es su carácter de fuertemente disciplinar procedimientos y mecanicismos de acción. Básicamente, tal legislación limitase a definir los documentos a ser presentados y los mecanicismos de su tramitación en los órganos de la administración estadual. Ejemplos de ese tipo de legislación son presentados en la Tabla 1.

Tabla 1

EJEMPLOS DE LEGISLACIÓN DE ESTADOS BRASILEÑOS Y SUS APLICACIONES

\begin{tabular}{|c|l|}
\hline Legislación/ Estado & \multicolumn{1}{|c|}{ Áreas de Aplicación o Reglamentación } \\
\hline $\begin{array}{c}\text { Decreto 3.910/81 } \\
\text { Rio de Jareiro }\end{array}$ & $\begin{array}{l}\text { Establece norma para el examen y anuencia de proyectos de } \\
\text { parcelamientos y desmembraciones en el Estado. }\end{array}$ \\
\hline $\begin{array}{c}\text { Ley 6.063/82 } \\
\text { Santa Catarina }\end{array}$ & $\begin{array}{l}\text { Define condiciones en la que es prohibida la parcelación } \\
\text { del suelo y establece competencias para juzgamiento de } \\
\text { proyectos. }\end{array}$ \\
\hline $\begin{array}{c}\text { Ley 1.130/87 } \\
\text { Rio de Janeiro }\end{array}$ & $\begin{array}{l}\text { Define áreas de interés especial con áreas superior a 1.000.000 } \\
\mathrm{m}^{2} \text { y en regiones limítrofes de municipios. }\end{array}$ \\
\hline $\begin{array}{c}\text { Decreto 9.760/87 } \\
\text { Rio de Janeiro }\end{array}$ & $\begin{array}{l}\text { Define las normas de ocupación para proyectos de parcelación } \\
\text { en Áreas de Interés Especial. }\end{array}$ \\
\hline $\begin{array}{c}\text { Decreto 47.817/2003 } \\
\text { São Paulo }\end{array}$ & $\begin{array}{l}\text { Define como competencia de la Secretaria de la Habitación el } \\
\text { examen y anuencia previa de propuestas de parcelación. }\end{array}$ \\
\hline $\begin{array}{c}\text { Resolución SH-17/2004 } \\
\text { São Paulo }\end{array}$ & $\begin{array}{l}\text { Define principios para la regularización de parcelación del } \\
\text { suelo anterior a la Ley 6.766/79. }\end{array}$ \\
\hline
\end{tabular}

En la implantación de estas propuestas de reglamentación, los estados brasileños tienen definidos una serie de documentos y procedimientos, por veces bastante abarcables y complejos, para las actividades de licenciamiento de nuevas parcelaciones urbanas. 
En el caso del Estado de Rio de Janeiro, por ejemplo, el instrumento utilizado para hacerse cumplir las definiciones del Decreto 3.910/81 es el Término de Referencia para presentación de Informe de Control Ambiental Simplista (RCAS).

El informe debe contener: situación y localización; articulación con unidades de conservación vecinas; propuesta urbanística, condiciones de relevo, geológicas y geotécnicas; alteraciones en la vegetación, propuesta de arborizar y recomponer la vegetación; áreas de préstamos y basurero; infraestructura y servicios urbanos; abastecimiento y drenaje de aguas; residuos sólidos y líquidos e contener un pronóstico de los impactos y de medidas que mitiguen y compensen previamente.

Otro estado que tiene reglamentación vasta acerca del asunto es el Estado de São Paulo. La estrategia fue adoptar la creación de un grupo denominado GRAPROHAB (Grupo de Análisis y Aprobación de Proyectos Habitacionales del Estado de São Paulo) para centralizar y agilizar los procedimientos de anuencia previa del Estado para proyectos de parcelación del suelo urbano. (GRAPROHAB, 2003)

Tal grupo estableció el conjunto de documentaciones a ser presentados para el análisis, para atender las exigencias legales y operacionales de las siguientes secretarias y órganos: CETEB (prevención y control de contaminación ambiental); Secretaria de la Habitación (anuencia de proyectos); Procuraduría General del Estado (documentación de propiedad); Secretaria del Medio Ambiente (legislación ambiental); Distribuidoras de Energía, Agua y Gas (adecuación a la infraestructura); Bomberos (sistemas de seguridad y protección contra incendio); Emplasa (adecuación a la planificación urbanística).

Un aspecto positivo de tal propuesta es el ancho espectro de la legislación considerada por el conjunto de los órganos que evalúen el proyecto, como se puede verificar en la Tabla 2.

\subsubsection{Tratamiento del Asunto en la Legislación de los Municipios}

Cuanto a la elaboración del Plano Director del Municipio, se nota la existencia de tres realidades en Brasil: municipios de menor porte no elaboran planes directores; en los municipios de portes medianos, la falta de equipo técnico hace con que los planes sean muy limitados; y en municipios de gran porte, que cuentan con un equipo técnico y recursos, estos planes realmente presentan avances en la legislación ambiental.

En los municipios de porte medianos, los planes directores se limitan a tratar de los impactos consecuentes de propuestas de implantación de nuevas parcelaciones del suelo reproduciendo las restricciones existentes en la legislación federal. Tal situación puede ser ilustrada con los planos directores de Torres (PMT, 1996), Londrina (PML, 1998), Sorocaba (PMS, 2004) y Viçosa (PMV, 2004).

En los municipios de gran porte, en especial capitales y regiones metropolitanas, la más grande capacitación del equipo técnico, asociada a la más grande complejidad de los problemas ambientales, hacen con que los instrumentos legales sean más completos y sofisticados. En algunos casos, planos directores ya habían previsto la realización de estudios de impacto de vecindad, anteriormente a la Ley 10.257, en otros los planos directores contemplan otra modalidad de evaluación de impactos en áreas urbanas.

El Plano Director de Fortaleza (PMF, 1992) prevé que emprendimientos que puedan representar excepcional sobrecarga en la capacidad de infraestructura del municipio deben pasar por análisis específica antes de su autorización.

En João Pessoa, el Plano Director (PMJP, 1993) prevé que propuestas de uso y ocupación del suelo que vengan a sobrecargar la infraestructura urbana, la red viaria y de trasportes, presenten estudios que comprueben su adecuación a las condiciones existentes, y indica el Estudio de Impacto de Vecindad para tal finalidad. 
Tabla 2

DOCUMENTOS SOLICITADOS POR LOS ÓRGANOS Y EMPRESAS QUE COMPONEN EL SISTEMA DE ANUENCIA PREVIA PARA PARCELAMIENTOS DEL GRAPROHAB (2002)

\begin{tabular}{|c|c|c|c|c|c|c|c|}
\hline \multirow{2}{*}{ Instrumento } & \multicolumn{7}{|c|}{ Órgano Responsable por la Evaluación } \\
\hline & SH & SMA & PGE & Cetesb & Sabesp & Emplasa & Bomberos \\
\hline Ley $6.766 / 79$ & $\mathrm{X}$ & $\mathrm{X}$ & $\mathrm{X}$ & $\mathrm{X}$ & & $\mathrm{X}$ & \\
\hline Ley $9.785 / 99$ & $\mathrm{X}$ & $\mathrm{X}$ & $\mathrm{X}$ & $\mathrm{X}$ & & $\mathrm{X}$ & \\
\hline Ley $898 / 75$ & $\mathrm{X}$ & $\mathrm{X}$ & & $\mathrm{X}$ & & & \\
\hline Ley $1.172 / 7$ & $\mathrm{X}$ & $\mathrm{X}$ & & $\mathrm{X}$ & & & \\
\hline Ley $2.177 / 79$ & & $\mathrm{X}$ & & $X$ & & & \\
\hline Decreto $9.714 / 77$ & & $\mathrm{X}$ & & $\mathrm{X}$ & & & \\
\hline Ley $4.056 / 84$ & $X$ & $\mathrm{X}$ & & $\mathrm{X}$ & & & \\
\hline Decreto $34.542 / 92$ & $\mathrm{X}$ & & & & & & \\
\hline Res. SNM 036/81 & $X$ & & & & & & \\
\hline Res. SH 039/97 & $\mathrm{X}$ & & & & & & \\
\hline Res. SH 069/92 & $\mathrm{X}$ & & & & & & \\
\hline Ley $997 / 76$ & & & & $\mathrm{X}$ & & & \\
\hline Decreto $8.468 / 76$ & & & & $\mathrm{X}$ & & & \\
\hline Decreto $10.229 / 77$ & & & & $\mathrm{X}$ & & & \\
\hline Ley $4.771 / 65$ & & $\mathrm{X}$ & & & & & \\
\hline Ley $7.803 / 89$ & & $\mathrm{X}$ & & & & & \\
\hline Decreto $750 / 93$ & & $\mathrm{X}$ & & & & & \\
\hline Ley $5.597 / 87$ & & $X$ & & & & & \\
\hline Res. CONAMA 001/86 & & $\mathrm{X}$ & & & & & \\
\hline Res. CONAMA 011/86 & & $\mathrm{X}$ & & & & & \\
\hline Res. CONAMA 002/88 & & $\mathrm{X}$ & & & & & \\
\hline Res. CONAMA 010/88 & & $\mathrm{X}$ & & & & & \\
\hline Res. CONAMA 004/93 & & $\mathrm{X}$ & & & & & \\
\hline Res. CONAMA 010/93 & & $\mathrm{X}$ & & & & & \\
\hline Res. CONAMA 001/94 & & $\mathrm{X}$ & & & & & \\
\hline Res. CONAMA 002/96 & & $\mathrm{X}$ & & & & & \\
\hline Res. CONAMA 003/96 & & $\mathrm{X}$ & & & & & \\
\hline Res. CONAMA 007/96 & & $\mathrm{X}$ & & & & & \\
\hline
\end{tabular}




\begin{tabular}{|c|c|c|c|c|c|c|c|}
\hline Res. SMA 42/94 & & $\mathrm{X}$ & & & & & \\
\hline Res. SMA 55/95 & & $\mathrm{X}$ & & & & & \\
\hline Res. SMA 16/2001 & & $\mathrm{X}$ & & & & & \\
\hline Res. SMA 21/2001 & & $\mathrm{X}$ & & & & & \\
\hline Res. SMA/ IBAMA 001/94 & & $\mathrm{X}$ & & & & & \\
\hline Res. SMA/ IBAMA 002/94 & $\mathrm{X}$ & $\mathrm{X}$ & & & & & \\
\hline Res. SMA/ IBAMA 005/96 & & $\mathrm{X}$ & & & & & \\
\hline Port. DEPRN 17/98 & & $\mathrm{X}$ & & & & & \\
\hline Port. DEPRN 42/2000 & & $\mathrm{X}$ & & & & & \\
\hline Decreto 46.076/2001 & & & & & & & $\mathrm{X}$ \\
\hline Port. DAEE 717/96 & & & & $\mathrm{X}$ & $\mathrm{X}$ & & \\
\hline NBR 7.229 & & & & $\mathrm{X}$ & $\mathrm{X}$ & & \\
\hline NBR 13.969 & & & & $\mathrm{X}$ & $\mathrm{X}$ & & \\
\hline NBR 12.208 & & & & $\mathrm{X}$ & $\mathrm{X}$ & & \\
\hline NBR 8.160/9.648/9.649 & & & & & $\mathrm{X}$ & & \\
\hline NB 568/569/570 & & & & & $\mathrm{X}$ & & \\
\hline NB 587/588/589 & & & & & $\mathrm{X}$ & & \\
\hline NB 591/592/593/594 & & & & & $\mathrm{X}$ & & \\
\hline NBR 13.103/13.525 & & & & & & & $\mathrm{X}$ \\
\hline
\end{tabular}

Port. Es una documentación oficial de acto administrativo, hecha por autoridades públicas y destinada a dar instrucciones o hacer determinaciones de varias órdenes.

En el municipio de Rio de Janeiro indica el uso de los Estudios de Impactos de Vecindad y los distingue de los Estudios de los Impactos Ambientales sin, entretanto, establecer los criterios claros de diferenciaciones entre ellos (PMRJ, 2001). En Porto Alegre (PMPA, 2004), el Plan Director define como Emprendimiento Puntual, con necesidad de Proyecto Especial, las propuestas de parcelación del suelo urbano, que deben presentar Estudios de Viabilidad Urbanística para tales actuaciones.

El Plan Director de São Paulo (PMSP, 2004) exige el licenciamiento de actividades que utilicen recursos ambientales, efectiva o potencialmente contaminadoras, o capaces de causar degradación ambiental, y distingue las actividades de los cuales serán exigidos Estudios de Impacto Ambiental, de aquellas de las cuales se exigirá Estudios de Impacto de Vecindad.

Un aspecto interesante a destacarse con relación a los planes directores es que, en cumplimiento a la Ley 10.257/2001, 2.353 municipios brasileños están obligados a elaborar o revisar sus planes directores. De estos, cerca de 1.700 tienen hasta octubre de 2006 para hacerlo (Ministerio de las Ciudades, 2004). 
Además de los planos directores, la necesidad de estudios ambientales para posibilitar propuestas de ocupación en áreas urbanas ha obtenido reglamentación en legislación correlato o complementar.

La ciudad de Natal (PMN, 1995) posee legislación específica que trata de la elaboración de RIV, como Ley Complementaria del Plano Director de la Ciudad, la cual se define los principio y el contenido mínimo de tales estudios. La Ley 1869/98 (Distrito Federal, 1998) del Distrito Federal, prevé el Informe de Impacto de Vecindad como uno de los instrumentos de evaluación de impacto ambiental.

En Anápolis (PMA, 2002), legislación específica de protección al patrimonio histórico y cultural de la ciudad prevé la realización de EIV visando la protección del paisaje urbano y del patrimonio histórico y cultural.

En Niteroi la Ley 2.050/2003 (PMN, 2003) establece las actividades que dependerán de la elaboración de EIV/ RIV, cuanto a su naturaleza, área y funcionamiento, define los parámetros de análisis a ser adoptados y el contenido mínimo del estudio.

\section{Ley 10.257 y sus implicaciones}

Todas las informaciones presentadas hasta este momento apuntan para la realidad de que los municipios son los grandes interesados, $\mathrm{y}$, por lo tanto los grandes responsables por la legislación que venga a regir el asunto.

A pesar del Impacto de Vecindad ser un instrumento de gestión ambiental urbana ya discutido en el medio técnico brasileño desde el inicio de la década de 90 , su conocimiento por los órganos del poder público municipal solamente se concretó con la promulgación de la Ley 10.257/2001 (Brasil, 2001).

\subsection{Principios y Dispositivos de la Ley 10.257}

Para Moreira (1999), siendo el medio ambiente entendido como relaciones de los hombres con la naturaleza para preservación de los recursos naturales en procesos de desarrollo sustentable (visión de la Comisión Bruntland 1998) el ambiente urbano puede ser entendido como conjunto de relaciones de los hombres con el espacio construido y la naturaleza. Desequilibrios ocurridos en el conjunto caracterizarían impactos ambientales.

En esa visión, el término vecindad puede ser entendido como la parcela del terreno sujeto al impacto en análisis. A pesar de ese concepto simple, el significado espacial del termo vecindad debe ser regido con relativa flexibilidad, ya que su delimitación depende del emprendimiento en análisis y del impacto considerado.

El objetivo de la Ley 10.257 fue reglamentar los artículos 182 y 183 de la Constitución Brasileña. Entre los asuntos tratados se tiene el Estudio de Impacto de Vecindad como instrumento de evaluación de propuestas de ocupaciones y actividades urbanas (Art. 36 hasta 38).

El EIV vino a cubrir un vacío en la legislación brasileña en lo que respecta a la evaluación de impactos ambientales consecuentes de la ocupación urbana por emprendimiento no tratados por las resoluciones del CONAMA, que exigen la realización de Estudios de Impacto Ambiental y producción de Informes de Impacto Ambiental (Mata, 2004).

La Ley 10.257 preserva el principio constitucional al prever la competencia de la unión en legislar cuanto a las normas generales, y da a la legislación municipal el poder de definir la forma de aplicación de los principios e instrumentos previstos. La definición del municipio como responsable por la legislación ambiental urbana crea la condición para que cada municipio trate las cuestiones ambientales según sus necesidades (Guimarães, 2004). 
Para la Ley 10.257, el EIV debe incluir «el análisis, en el mínimo, de las siguientes cuestiones»: concentración de la población; equipamientos urbanos y comunitarios; uso y ocupación del suelo; ventilación e iluminación; paisaje urbano y patrimonio natural y cultural.

La definición de los componentes ambientales es una de las deficiencias de la Ley. Tales análisis no se pueden restringir al sistema viario, las características urbanísticas y disponibilidad de infraestructura. Impactos sobre los recursos naturales, emisión de ruidos, emanación de gases y vapores e generación de residuos se deben llevar en consideración.

\subsection{Propuestas de Ampliación de los Componentes Evaluados}

A consecuencia del reconocimiento de las deficiencias de la Ley 10.257, se propone un conjunto más amplio de componentes ambientales a ser considerados en EIV, de forma que considere todos los impactos de vecindad posibles en propuestas de parcelamientos o conjuntos residenciales.

Con el objetivo de estructurar mejor la identificación de los impactos, estos fueron agrupados en cuatro categorías: impactos en el medio físico; impactos urbanísticos; impactos en la infraestructura urbana; e impactos en el saneamiento y calidad de vida.

En el grupo medio físico los componentes son: aire, suelos, rocas, relieve, manantiales superficiales y subterráneos, paisaje natural, vegetación, uso y ocupación del suelo.

Los componentes definidos para la categoría «impactos urbanísticos» son: densidad de población, densidad urbana, mercado inmobiliario, ventilación, iluminación, paisaje urbano, patrimonio cultural, calidad urbanística.

El grupo «infraestructura urbana» incluye los componentes de servicios y materias primas: vías urbanas, transporte colectivo urbano, redes de agua, alcantarilla, drenaje pluvial, energía eléctrica, telefonía, iluminación pública y seguridad pública.

En la categoría denominada «Saneamiento y Calidad de Vida» se encuentran los componentes: ruidos, residuos sólidos urbanos, residuos líquidos urbanos, y residuos industriales.

\section{Problemas existentes en la legislación}

A pesar de la cantidad de leyes, decretos, y resoluciones aquí citados, varias deficiencias en el uso de tales instrumentos de gestión han sido notados. En la mayoría de los casos tales deficiencias tienen más relación con la legislación que con su fiscalidad. Los problemas son vastos, pero pueden ser divididos en tres categorías, en función de su naturaleza: problemas de carácter filosófico; de carácter técnico; y de carácter operacional.

De facto los problemas presentan una relación de dependencia clara: los filosóficos causan los técnicos, los cuales, por su vez, condicionan los problemas operacionales. Una visión equivocada en la legislación induce la adopción de criterios técnicos deficientes o de difícil aplicación, los cuales causan dificultades operacionales.

\subsection{De Carácter Filosófico}

Para Azevedo Neto (1999) uno de los principales problemas son los presupuestos equivocados adoptados en la planificación. Entre ellos el autor destaca el presupuesto de que la ciudad se construye lote a lote. De facto la tendencia actual es de construcción de grandes emprendimientos, «Shopping Centers» (centros comerciales), centros empresariales, conjuntos residenciales, condominios industriales, agregando pequeños lotes preexistentes. 
Por otro lado, la secuencia clásica de primero aparecer el parcelamiento y después las construcciones individuales también están siendo conturbada por la construcción de grandes condominios, sin pasar por la fase de conjunto de lotes.

Costa \& Braga (2002) citan la falta de vínculo entre política ambiental y las demasiadas políticas públicas, con la acción del órgano ambiental generalmente restricta a la gestión de espacios verdes y fiscalización de fuentes fijas de polución. Con eso, cuestiones vueltas a la política ambiental, como saneamiento, sistema de transportes y uso y ocupación del suelo, son tratadas por otros órganos, sin integración y con actuación puntual.

Maricato (2003) afirma que mismo con el proceso de urbanización en Brasil dándose en el siglo XX, él fue contaminado por características de los periodos colonial e imperial, como concentración de tierra, renta y poder, y aplicación arbitraria de la ley.

La autora acuerda que la legislación urbana solamente surgió cuando se hizo necesaria para la estructuración del mercado inmobiliario, y que históricamente los Códigos Municipales de Posturas, tuvieron un papel de subordinar ciertas áreas de la ciudad al capital inmobiliario acarreando la expulsión de la masa trabajadora pobre del centro de la ciudad.

Tucci (2003), evaluando la filosofía de proyectos de drenaje urbano, resalta que los proyectos no tratan la bacía como un todo, no se integran con el Plano Director, y no consideran el control de los efluentes de forma integrada al drenaje.

\subsection{De Carácter Técnico}

Freitas (2001) enseña ejemplos de alteraciones ambientales debido a la implantación de emprendimientos habitacionales (Tabla 3), ni siempre son tratadas por la legislación.

Con relación a la protección de la vegetación, definición de áreas de preservación, Araújo (2002) destaca que para las áreas urbanas, la legislación remite la definición de criterios a los planes directores y leyes municipales de uso del suelo. Dada la falta o deficiencia de tal legislación, las áreas de preservación permanente son objeto constante de modificaciones.

Al discutir como cambiar la realidad (ya descrita) de la limitación de los proyectos de drenaje, en termos de su pequeño alcance ambiental, Tucci (2003) sugiere que la legislación para futuros emprendimientos sea incorporada al Plano Director.

Silva (2004) acuerda que entre los años de 1964 y 1986 el sistema financiero de la habitación consideraba dos padrones de edificaciones: uno de residencias más grandes y más individualizadas próximas a la área central, y otro de residencias más pequeñas y muy agrupadas en las porciones periféricas. Esa tendencia de conjuntos habitacionales agrupados en las periferias, además de crear áreas de pobreza, trajo serios problemas de orden urbanística, relacionados a la red viaria y de transporte urbano.

Al discutir la cuestión de los llamados «condominios urbanísticos» (los condominios cerrados), Araújo (2004) enseña que la ley de los condominios e incorporaciones contiene un permiso genérico para implantación de esas iniciativas, sin cualquier exigencia urbanística o ambiental.

Así, exactamente por no ser claramente entendido por la ley como una modalidad de parcelación del suelo urbano, el condominio urbanístico ha sido usado como una forma de escape a las exigencias urbanísticas y ambientales derivadas de la Ley 6.766/79 y de la Resolución CONAMA 01/86. 
Tabla 3

EJEMPLOS DE ALTERACIONES AMBIENTALES EN CONSECUENCIA DE

EMPRENDIMIENTO CON FUNCIÓN HABITACIONAL, SEGÚN FREITAS ET.AL.

(2001)

\begin{tabular}{|c|l|}
\hline Seguimiento Ambiental & \multicolumn{1}{|c|}{ Alteración } \\
\hline \multirow{3}{*}{ Medio Físico } & Aceleración del proceso erosivo; \\
& Ocurrencia de escorrentías; \\
& Aumento de áreas con inundación; \\
& Disminución de la Infiltración de agua en el suelo; \\
& Contaminación del suelo y aguas superficiales y subterrá- \\
& neas; \\
& Aumento de partículas y gases en la atmósfera; \\
& Aumento de ruidos. \\
\hline \multirow{5}{*}{ Medio Biótico } & Supresión de la vegetación; \\
& Degradación de la vegetación por el efecto de borda; \\
& Degradación de la vegetación por deposición de partículas; \\
& Daños a la fauna; \\
& Incómodos a la fauna. \\
\hline \multirow{5}{*}{ Medio Socioeconómico } & Aumento de la demanda por servicios públicos; \\
& Aumento del consumo de agua y energía; \\
& Aumento de las operaciones comerciales; \\
& Aumento de la recaudación de impuestos; \\
& Aumento de oferta de empleos; \\
& Aumento en el tráfago; \\
& Alteración en la percepción ambiental; \\
& Cambios de referencias culturales. \\
\hline
\end{tabular}

\subsection{De Carácter Operacional}

Duarte (2001) destaca que los ritos burocráticos creado por el poder público complican las cosas porque: la documentación necesita tramitar por varios órganos; ni todos los órganos tienen conocimientos de los criterios adoptados, el control sobre la documentación es limitado y difícil, el control sobre los contenidos de los procesos también, no hay integración entre los sectores generando actitudes incorrectas.

Con relación a los problemas de carácter operacional, Costa \& Braga (2002) resaltan que la desvinculación de la política ambiental brasileña de las demasiadas políticas públicas no se resume a la simple falta de integración, comprende también, y principalmente, la existencia de objetivos y lógicas contradictorias entre las diferentes políticas formuladas e implementadas por el poder público municipal.

Destacan todavía que es frecuente que la ocurrencia de conflictos entre la agencia ambiental y otros órganos del poder municipal responsables por la realización de obras públicas. La propia administración municipal lleva a cabo acciones de gran impacto sobre el medio ambiente (obras viarias, canalización de riachuelos y ríos, implantación de terraplén sanitarios y depósitos de residuos). 


\section{Componentes e impacto a seren evaluados}

La lista de componentes ambientales susceptibles de alteración y de degradación por parcelamientos es muy vasta y la interacción entre tales componentes bastante compleja. Además de eso, la forma como tales alteraciones se procesan y los componentes ambientales afectados, dependen de la fase de la actuación que se considere.

En el caso de parcelamientos y conjuntos habitacionales dos fases importantes deben ser consideradas, la fase de implantación y la de operación. Para destacar los impactos característicos de cada fase, en función de la intervención y de los componentes afectados, el trabajo presenta cada una de esas fases separadamente.

Tabla 4

INTERVENCIONES PREVISTAS, IMPACTOS ESPERADOS Y COMPONENTES AMBIENTALES AFECTADOS EN LA FASE DE IMPLANTACIÓN DE PARCELAMIENTO O CONJUNTO HABITACIONAL

\begin{tabular}{|c|c|c|}
\hline Intervención & Impactos Esperados & Componentes Ambientales Afectados \\
\hline $\begin{array}{l}\text { Creación de } \\
\text { accesos }\end{array}$ & $\begin{array}{l}\text { Deshacer la } \\
\text { caracterización de la área } \\
\text { y entorno }\end{array}$ & $\begin{array}{l}\text { Patrimonio Cultural (I), Calidad urbanística } \\
\text { (I), Vías Urbanas (D), Transporte Urbano (D), } \\
\text { Suelos (D), Rocas (I), Relevo (D), Manantial de } \\
\text { superficie (I), Manantial subterráneo (I), Paisaje } \\
\text { natural (I), Vegetación (I), Uso y Ocupación (I), } \\
\text { Nivel de ruido (I). }\end{array}$ \\
\hline $\begin{array}{l}\text { Adaptación de } \\
\text { accesos }\end{array}$ & $\begin{array}{l}\text { Deshacer la } \\
\text { caracterización de la área } \\
\text { y entorno }\end{array}$ & $\begin{array}{l}\text { Patrimonio Cultural (I), Calidad urbanística (I), } \\
\text { Vías Urbanas (I), Transporte Urbano(D), Uso y } \\
\text { Ocupación (I), Nivel de ruido (I). }\end{array}$ \\
\hline \multirow{3}{*}{ Terraplén } & $\begin{array}{l}\text { Movilización de } \\
\text { vehículos }\end{array}$ & $\begin{array}{l}\text { Vías Urbanas (D), Patrimonio Cultural (I), Aire } \\
\text { (D), Nivel de ruido (D). }\end{array}$ \\
\hline & Operación de máquinas & $\begin{array}{l}\text { Paisaje Urbana (I), Paisaje natural (I), Vías } \\
\text { Urbanas (D), Patrimonio Cultural (I), Aire (D), } \\
\text { Vegetación (I), Nivel de ruido (D). }\end{array}$ \\
\hline & $\begin{array}{l}\text { Operación de } \\
\text { equipamientos }\end{array}$ & $\begin{array}{l}\text { Paisaje Urbana (I), Patrimonio Cultural (I), Aire } \\
\text { (D), Nivel de ruido (D). }\end{array}$ \\
\hline \multirow[t]{2}{*}{$\begin{array}{l}\text { Instalación del } \\
\text { cantero de obras }\end{array}$} & $\begin{array}{l}\text { Conexión a la } \\
\text { infraestructura urbana }\end{array}$ & $\begin{array}{l}\text { Red de agua (D), Red de Alcantarilla (D), Red } \\
\text { de drenaje pluvial (D), Red eléctrica (D), Red } \\
\text { telefónica (I), Red de iluminación pública (I), } \\
\text { Suelos (I), Rocas (I), Manantial de superficie (I), } \\
\text { Manantial subterráneo (I), Nivel de ruido (D). }\end{array}$ \\
\hline & $\begin{array}{l}\text { Transporte y almacenaje } \\
\text { de materiales de } \\
\text { construcción }\end{array}$ & $\begin{array}{l}\text { Vías Urbanas (I), Suelos (D), Rocas (I), Manantial } \\
\text { de superficie (D), Manantial subterráneo (D), } \\
\text { Vegetación (I), Residuos sólidos urbanos (I). }\end{array}$ \\
\hline $\begin{array}{l}\text { Obras de } \\
\text { construcción, } \\
\text { drenaje y } \\
\text { pavimentación. }\end{array}$ & $\begin{array}{l}\text { Excavaciones, generación } \\
\text { de residuos de } \\
\text { construcción. }\end{array}$ & $\begin{array}{l}\text { Vías Urbanas (D), Red de agua (I), Red de } \\
\text { alcantarilla (I), Red de drenaje pluvial (I), Suelos } \\
\text { (D), Rocas (D), Manantial de superficie (I), } \\
\text { Manantial subterráneo (I), Residuos sólidos } \\
\text { urbanos (D). }\end{array}$ \\
\hline
\end{tabular}


Tabla 5

INTERVENCIONES PREVISTAS, IMPACTOS ESPERADOS Y COMPONENTES AMBIENTALES AFECTADOS EN LA FASE DE OPERACIÓN DEL PARCELAMIENTO O CONJUNTO HABITACIONAL

\begin{tabular}{|c|c|c|}
\hline Intervención & Impactos Esperados & Componentes Ambientales Afectados \\
\hline \multirow{2}{*}{$\begin{array}{l}\text { Demanda por } \\
\text { insumos e } \\
\text { infraestructura }\end{array}$} & $\begin{array}{l}\text { Movilización de } \\
\text { vehículos }\end{array}$ & $\begin{array}{l}\text { Vías Urbanas (D), Patrimonio Cultural (I), Aire } \\
\text { (D), Nivel de ruido (D). }\end{array}$ \\
\hline & Gerencia de materiales & $\begin{array}{l}\text { Red de Alcantarilla (I), Red eléctrica (I), Red } \\
\text { telefónica (I), Iluminación pública (I), Seguridad } \\
\text { pública (I), Transformaciones urbanísticas (I). }\end{array}$ \\
\hline $\begin{array}{l}\text { Entrada de } \\
\text { materias primas }\end{array}$ & Circulación de materiales & $\begin{array}{l}\text { Aire (D), Paisaje natural (I), Vías Urbanas } \\
\text { (I), Seguridad pública (I), Transformaciones } \\
\text { urbanísticas (I), Ruido (D). }\end{array}$ \\
\hline $\begin{array}{l}\text { Circulación de } \\
\text { personal }\end{array}$ & $\begin{array}{l}\text { Solicitudes en la } \\
\text { infraestructura y } \\
\text { servicios públicos }\end{array}$ & $\begin{array}{l}\text { Vías Urbanas (D), Transporte Urbano(D), } \\
\text { Iluminación pública (D), Seguridad pública (D) }\end{array}$ \\
\hline \multirow{3}{*}{$\begin{array}{l}\text { Adaptaciones } \\
\text { y ampliaciones } \\
\text { de obras o } \\
\text { servicios. }\end{array}$} & $\begin{array}{l}\text { Movilización de } \\
\text { vehículos }\end{array}$ & $\begin{array}{l}\text { Vías Urbanas (D), Patrimonio Cultural (I), Aire } \\
\text { (D), Nivel de ruido (D). }\end{array}$ \\
\hline & Operación de máquinas & $\begin{array}{c}\text { Paisaje urbana (I), Paisaje natural (I), Vías } \\
\text { Urbanas (D), Patrimonio Cultural (I), Aire (D), } \\
\text { Vegetación (I), Nivel de ruido (D). }\end{array}$ \\
\hline & $\begin{array}{l}\text { Operación de } \\
\text { equipamientos }\end{array}$ & $\begin{array}{l}\text { Paisaje Urbanas (I), Patrimonio Cultural (I), } \\
\text { Aire (D), Nivel de ruido (D). }\end{array}$ \\
\hline
\end{tabular}

Es fundamental que se destaque que se puede haber una superposición entre las fases, en parte del emprendimiento las actividades de implantación pueden estar concluidas y las operaciones urbanas dándose plenamente, en cuanto en otra parcela las actividades se encuentran en fase de implantación, o en los dos casos pueden estar en desarrollo concomitante. Las Tablas 4 y 5 presentan las intervenciones, los impactos, y los componentes afectados en las fases de implantación y operación (respectivamente).

Los índices I y D, presentados entre paréntesis, indican la relación causal entre impacto y componente ambiental, donde I representa el impacto indirecto y $\mathrm{D}$, impacto directo.

\section{Técnicas y procedimientos para evaluación}

Cuanto a los procesos de evaluación de impactos de vecindad, algunos cuidados deben ser tomados. Tales cuidados se justifican en función de deficiencias existentes en la legislación y en las técnicas de análisis más comunes.

Las deficiencias legales incluyen imprecisiones o limitaciones de la Ley 10.257/2001 o de las leyes municipales que definan o vengan a definir los componentes ambientales a ser evaluados en EIV.

El artículo 37 de la Ley 10.257/2001 establece que «el EIV será ejecutado de forma a contemplar los efectos positivos y negativos del emprendimiento o actividad cuanto a la calidad de vida de la población residente en el área y sus proximidades, incluyendo el análisis, en el mínimo, de las siguientes cuestiones...». 
La ley usa ese tipo de redacción porque desempeña un papel amplio de reglamentación y remite a la legislación municipal a la definición precisa de tales componentes.

Sin embargo, cuando de la proposición de leyes municipales la expresión «en el mínimo» acostumbra ser considerada (por incapacidad o conveniencia), quedando las legislaciones municipales restrictas a los tópicos propuestos en la ley federal.

Con relación a las cuestiones a la que se refiere el artículo, el texto es superficial y vacío con relación a algunos factores ambientales, tales como equipamientos urbanos y comunitarios y, especialmente, paisaje urbana y patrimonio natural y cultural.

Con relación a los «equipamientos urbanos y comunitarios» debe ser dada especial atención a la diversidad de dispositivos de infraestructura que pueden sufrir impactos, tales como: vías públicas; redes públicas de abastecimiento de agua y drenaje urbana; disponibilidad de servicios particulares; cuestiones relacionadas a la seguridad; y demanda por aparcamiento.

Eso no suele ocurrir. Lo que se observa generalmente es que apenas una parte de tales factores es considerada, especialmente en lo que dice respecto a vías públicas, redes de agua y drenaje urbana y aparcamiento.

En lo que dice respecto al paisaje urbano y patrimonio natural y cultural, es común que las leyes municipales y, por consecuencia, los estudios de ellas consecuentes consideren apenas el patrimonio histórico y artístico y aspectos paisajísticos, no valorando debidamente la protección de componentes del medio físico.

Así, la protección de recursos naturales tales como manantiales subterráneos y de superficie, suelos, rocas y vegetación, no acostumbra ser considerada ni del punto de vista de su degradación física, como de su contaminación y polución. En un momento, tales bienes son de gran importancia para calidad ambiental, y no podrían ser despreciados en estudios de impactos de vecindad.

En cuanto a las deficiencias en las técnicas de análisis, los presupuestos asumidos en el inicio de un Estudio de Impacto de Vecindad, en lo que dice respecto a los factores a ser evaluados y a las características del emprendimiento y de la vecindad son determinantes en el suceso del trabajo.

Incluso con los presupuestos adecuados, la forma de conducir los análisis también es determinante para que se obtenga resultados fidedignos. El levantamiento de los datos determina la calidad de la información a ser tratada, y la seguridad en el análisis define lo cuanto son confiables los resultados para fines de alternativas y medidas mitigadoras y compensatorias.

El emprendimiento cuyos impactos de vecindad a ser evaluado necesita ser muy bien caracterizado cuanto a su naturaleza, su porte, y propuesta de ocupación. La caracterización de la vecindad debe complementar sus condiciones ambientales actuales (base para previsión de impactos), y definición de dimensiones compatibles con el área de influencia del emprendimiento.

Las dimensiones de la vecindad (área de influencia) deben ser adaptadas de acuerdo con características del emprendimiento y del componente ambiental considerado.

En el caso de la construcción de un «Shopping Center» (Centro Comercial), por ejemplo, los impactos en el medio natural pueden limitarse a el área de construcción del mismo (menor área de influencia), pero los impactos en el tráfago urbano se pueden extender además de las vías vecinas a el emprendimiento englobando vías y acceso hasta su conexión con el sistema viario principal (aumentado significativamente el área de influencia).

La elaboración de Estudios de Impactos de Vecindad que no describen o no evalúan apropiadamente las condiciones del emprendimiento, de la vecindad, o los componentes 
ambientales, genera consecuencias malas en cuatro esferas, a saber: para el ambiente, para la población vecina, para la población en general, y para el poder público.

Para el ambiente las consecuencias dañosas incluyen todas las formas de degradación, contaminación o polución que vengan a perjudicar la calidad de los componentes ambientales.

Tales efectos pueden hacerse sentir localmente (en la vecindad considerada) o pueden, si no debidamente vigilados y tratados, extrapolar tales límites comprometiendo la calidad ambiental de otras áreas.

Eso es particularmente verdad en lo que se refiere a la contaminación o polución del aire y de aguas (subterráneas o de superficie), ya que tales medios fluidos circulan llevando las substancias nocibles para áreas además de la vecindad del punto donde se dio su lanzamiento.

Para la población vecina, la falta de detección previa de los impactos impide la adopción de medidas de control, mitigación, o mismo compensación, exponiendo a la población a los efectos de las intervenciones provocadas por el emprendimiento.

Además de las confusiones obvias, tal situación puede crear en la población de la vecindad un clima de animosidad con relación al emprendimiento y al poder público que torna más difícil la gestión posterior de los problemas, pudiendo acarrear demandas judiciales que desgastan todavía más las relaciones entre los actores del proceso.

Al degradar una parcela del área urbana del municipio los impactos de vecindad ya son, de forma indirecta, un problema de toda la población. Al extrapolar los limites del área de influencia del emprendimiento, tales impactos alcanzan directamente otras parcelas del municipio.

Al exigir del poder público prioridad de recursos en actividades de corrección, tales efectos alcanzan el presupuesto del municipio haciendo inviables otras inversiones.

Para el poder público además de la degradación y desvaloración de parcelas del municipio, y de la necesidad de inversiones para corregir los impactos, la ausencia de identificación previa de impactos de vecindad significa la pérdida de la oportunidad de negociar inversiones en medidas compensatorias.

Los problemas en consecuencia de vacíos en la legislación pueden ser reducidos con mayores detalles, por parte de los legisladores municipales, de las exigencias legales en dos aspectos: cuanto a los tipos de actuaciones susceptibles de realización previa de Estudio de Impacto de Vecindad para obtener licencia para instalación; y cuanto a los factores a ser obligatoriamente considerados en la elaboración de tales estudios.

Tal iniciativa impediría que emprendimientos que representan potencial de generación de impactos de vecindad se quedaran afuera de las exigencias legales, y obligaría los estudios realizados a considerar factores actualmente despreciados o tratados de forma impropia.

Con relación al proceso de análisis de los impactos de vecindad, la consideración cuidadosa de las peculiaridades de cada emprendimiento evaluado y de la vecindad considerada, bien como de los límites de la misma, puede tornar el proceso más eficiente.

La consideración con base en leyes más detalladas o por iniciativa de los responsables por los estudios, de factores hoy despreciados o menospreciados también traerían grandes beneficios a los estudios de impacto de vecindad, enriqueciéndolos y facilitando el proceso de toma de decisiones a partir de ellos.

\section{Conclusiones}

Para tener una acción efectiva en la defensa del ambiente urbano de los municipios brasileños, los Estudios de Impacto de Vecindad deben basarse en legislación municipal bien elaborada y abarcable. 
En preferencia, tales principios pueden ser adoptados en el Plan Director del municipio o en leyes a él subordinadas de forma a posibilitar al poder publico municipal una gestión responsable y eficaz del problema y, a la población como un todo la garantía del derecho constitucional al medio ambiente equilibrado.

Estudios de Impacto de Vecindad mejor elaborados, conteniendo un diagnóstico más fiable de la cuestión, permitirán al poder público seguridad en la imposición de medidas correctivas y compensatorias, así como la exigencia de adopción de técnicas de vigilancia de los impactos.

\section{Referencias bibliográficas}

ARAÚJO, S.M.V.G. (2002): As áreas de preservação permanente e a questão urbana. Consultoria Legislativa - Câmara dos Deputados. Brasília. 12 pp.

ARAUUJO, S.M.V.G. (2004): Condomínios Urbanísticos. Consultoria Legislativa - Câmara dos Deputados. Brasília. 16 pp.

AZEVEDO NETTO, D.T. (1999): Problemas e desafios do controle do uso do solo. en O Município no Século XXI: cenários e perspectivas. Fundação Prefeito Faria Lima, São Paulo, pp. 257-274.

BOCCASUIS-SIQUERA, A. (2002): O Direito Ambiental na Legislação Brasileira - Um contributo para o resgate da História. en Rev. eletrônica Mestr. Educ. Ambient., vol 09. Disponível em < http://www.remea.furg.br/>.

BRASIL (1934): Decreto 24.643, de 10 de Julho de 1934. Decreta o Código de Água. Governo da República dos Estados Unidos do Brasil, disponível em <http://www. lei.adv.br/>.

BRASIL (1937): Decreto-lei 25 de 30 de novembro de 1937. Organiza a proteção ao Patrimônio Histórico e Artístico Nacional. Governo da República dos Estados Unidos do Brasil, disponível em <http://www. lei.adv.br/>.

BRASIL (1964): Lei 4.591 de 16 de dezembro de 1964. Dispõe sobre o condomínio em edificações, de uma ou mais imobiliárias. República Federativa do Brasil, disponível em <http://www6. senado.gov.br $/>$.

BRASIL (1965a): Lei 4.771 de 15 de setembro de 1965. Insitui o Novo Código Florestal. República Federativa do Brasil, disponível em <http://www.planalto.gov.br/ legisla.htm>.

BRASIL (1965b): Lei 4.778 de 22 de setembro de 1965. Dispõe sobre a obrigatoriedade de serem ouvidas as autoridades florestais na aprovação de planos de loteamento para venda de terrenos em prestações. República Federativa do Brasil, disponível em <http://www. lei.adv.br/>.

BRASIL (1967): Decreto-lei 271 de 28 de fevereiro de 1967. Dispõe sobre loteamento urbano, responsabilidade do loteador, concessão de uso e espaço aéreo e dá outras providências. República Federativa do Brasil, disponível em < http://www. lei.adv.br/>.

BRASIL (1979): Lei 6.766 de 19 de dezembro de 1979. Dispõe sobre o Parcelamento do Solo Urbano e dá outras providências. República Federativa do Brasil, disponível em <http://www. lei.adv.br/>.

BRASIL (1981a): Decreto 86.176 de 06 de julho de 1981. Regulamenta a Lei $n^{\circ} 6.513$, de 20 de dezembro de 1977, que dispõe sobre a criação de Áreas Especiais e de Locais de Interesse Turístico, e dá outras providencias. República Federativa do Brasil, disponível em <http://www. lei.adv.br/>.

BRASIL (1981b): Lei 6.938 de 31 de agosto de 1981. Dispõe sobre a Política Nacional do Meio Ambiente, seus fins e mecanismos de formulação e aplicação, e dá outras providências. República Federativa do Brasil, disponível em <http://www.planalto.gov. br/ legisla.htm $>$.

BRASIL (1990): Decreto 99.274 de 06 de julho de 1990. Regulamenta a Lei $n^{\circ}$ 6.902, de 27 de Abril de 1981, e a Lei $n^{\circ}$ 6.938, de 31 de Agosto de 1981, que dispõem, respectivamente, sobre a criação de Estações Ecológicas e Áreas de Proteção Ambiental e sobre a Política Nacional do Meio Ambiente, e dá outras providências. República Federativa do Brasil, disponível em $<\mathrm{http}: / / \mathrm{www}$. lei.adv.br/>.

BRASIL (1996): Decreto 1.922 de 05 de junho de 1996. Dispõe sobre o reconhecimento das Reservas Particulares do Patrimônio Natural e dá outras providências. República Federativa do Brasil, disponível em <http://www6.senado. gov.br / >. 
BRASIL (1997): Lei 9.433 de 08 de janeiro de 1997. Institui a Política Nacional de Recursos Hídricos, cria o Sistema Nacional de Gerenciamento de Recursos Hídricos, regulamenta o inciso XIX do art. 21 da Constituição Federal, e altera o art. $1^{\circ}$ da Lei $n^{\circ} 8.001$, de 13 de março de 1990, que modificou a Lei $n^{\circ} 7.990$, de 28 de dezembro de 1989. República Federativa do Brasil, disponível em < http://www.planalto.gov.br/ legisla.htm >.

BRASIL (1999): Lei 9.785 de 29 de janeiro de 1999. Altera o Decreto-Lei $n^{\circ} 3.365$, de 21 de junho de 1941 (desapropriação por utilidade pública) e as Leis $n^{\circ}$ 6.015, de 31 de dezembro de 1973 (registros públicos) e 6.766, de 19 de dezembro de 1979 (parcelamento do solo urbano). República Federativa do Brasil, disponível em <http://www. lei.adv.br/>.

CONAMA (1986): Resolução Conama 001 de 23 de janeiro de 1986. Diário Oficial da União de 17 de fevereiro de 1986, disponível em <http://www.mma.gov.br/port/ conama/>.

CONAMA (1987a): Resolução Conama 010 de 03 de dezembro de 1987. Diário Oficial da União de 18 de março de 1988, disponível em <http://www.mma.gov.br/ port/conama/>.

CONAMA (1987b): Resolução Conama 011 de 03 de dezembro de 1987. Diário Oficial da União de 18 de março de 1988, disponível em <http://www.mma.gov.br/ port/conama/>.

CONAMA (1988): Resolução Conama 005 de 15 de julho de 1988. Diário Oficial da União de 16 de novembro de 1988, disponível em <http://www.mma.gov.br/port/ conama/>.

CONAMA (1989): Resolução Conama 012 de 14 de setembro de 1989. Diário Oficial da União de 18 de dezembro de 1989, disponível em <http://www.mma.gov.br/port/ conama/>.

CONAMA (1990): Resolução Conama 013 de 06 de dezembro de 1990. Diário Oficial da União de 28 de dezembro de 1990, disponível em <http://www.mma.gov.br/port/ conama/>.

CONAMA (1993): Resolução Conama 004 de 31 de março de 1993. Diário Oficial da União de 08 de abril de 1993, disponível em <http://www.mma.gov.br/port/ conama/>.

CONAMA (1996): Resolução Conama 002 de 18 de abril de 1996. Diário Oficial da União de 27 de abril de 1996, disponível em <http://www.mma.gov.br/port/ conama/>.

CONAMA (1997): Resolução Conama 237 de 19 de dezembro de 1997. Diário Oficial da União de 07 de janeiro de 1998, disponível em <http://www.mma.gov.br/port/ conama/>.

CONAMA (2002a): Resolução Conama 302 de 20 de março de 2002. Diário Oficial da União de 13 de maio de 2002, disponível em <http://www.mma.gov.br/ port/conama/>.

CONAMA (2002b): Resolução Conama 303 de 20 de março de 2002. Diário Oficial da União de 13 de maio de 2002, disponível em <http://www.mma.gov.br/ port/conama/>.

CONAMA (2002c): Resolução Conama 307 de 05 de julho de 2002. Diário Oficial da União de 17 de julho de 2002, disponível em <http://www.mma.gov.br/ port/conama/>.

CONAMA (2003a): Resolução Conama 339 de 25 de fevereiro de 2003. Diário Oficial da União de 13 de novembro de 2003, disponível em <http://www.mma.gov.br/port/ conama/>.

CONAMA (2003b): Resolução Conama 341 de 25 de setembro de 2003. Diário Oficial da União de 03 de novembro de 2003, disponível em <http://www.mma.gov.br/port/ conama/>.

CONAMA (2004): Resolução Conama 347 de 10 de setembro de 2004. Diário Oficial da União de 13 de setembro de 2004, disponível em <http://www.mma.gov.br/port/ conama/>.

COSTA, H.S.M. \& BRAGA, T.M. (2002): «Entre a conciliação e o conflito: dilemas para o planejamento e a gestão urbana e ambiental». X Seminário sobre a Economia Mineira, Disponível em $<$ www.cedepar.ufmg.br/diamantina2002>.

DISTRITO FEDERAL (1998). Lei 1869, de 21 de Janeiro de 1998. Dispõe sobre os instrumentos de avaliação de impacto ambiental no Distrito Federal e dá outras providências. Disponível em $<$ http://www.estatudodacidade.com.br $>$.

DUARTE, L.H.M. (2001): Geoprocessamento aplicado à análise de potencial de ocupação urbana de Ouro Preto, MG. Universidade Federal de Minas Gerais, Belo Horizonte. 63 pp.

FREITAS, C.G.L. (2001): Habitação e meio ambiente - abordagem integrada em empreendimentos de interesse social. Habitare. São Paulo. Disponível em <http:// habitare.infohab.org.br/>.

GRAPROHAB (2003): Manual para Projetos de Loteamentos e Núcleos Habitacionais. Secretaria de Estado da Habitação - Governo do Estado de São Paulo. São Paulo. 89 pp.

MINISTÉRIO DAS CIDADES (2004): Conheça a situação dos municípios em relação à obrigatoriedade de elaborar Planos Diretores. Disponível em <http://www.cidades. gov.br/>. 
GUIMARÃES, J.P. (2004): Competência Constitucional dos Municípios em Matéria Ambiental. en O Direito Ambiental das Cidades. DP\&A, Rio de Janeiro, pp. 85-102.

MARICATO, E. (2003): Metrópole, legislação e desigualdade. en Estudos Avançados, vol 17. n. 48, pp. 151-166.

MATA, L.R. (2004): O Estatuto da Cidade à Luz do Direito Ambiental. en O Direito Ambiental das Cidades. DP\&A, Rio de Janeiro, pp. 103-142.

MOREIRA, A.C.M.L. (1999): Conceitos de ambiente e impacto ambiental aplicados ao meio urbano. Faculdade de Arquitetura e Urbanismo, Universidade de São Paulo. São Paulo, Disponível em: $<$ http://www.usp.br/fau/docentes/depprojeto/a_moreira/ producao/producao.htm>.

PMA (2002): Lei 1.215 de 23de Dezembro de 2002. Cria o Conselho Municipal do Patrimônio Histórico e Cultural de Anápolis. Disponível em <http://www. estatudodacidade.com.br>.

PMF (1992): Plano Diretor de Fortaleza - CE. Prefeitura Municipal de Fortaleza. Disponível em $<$ http://www.estatudodacidade.com.br $>$.

PMJP (1993): Plano Diretor do Município de João Pessoa - PB. Prefeitura Municipal de João Pessoa. Disponível em: <http://www.estatutodacidade.com.br/>.

PML (1998): Lei Municipal 7.482 de 21 de julho de 1998. Prefeitura Municipal de Londrina. Londrina (PR), 12 pp.

PMN (1995): Lei 4.619 de 28 de Abril de 1995. Dispõe sobre a regulamentação do procedimento para análise do Relatório de Impacto de Vizinhança - RIV, conforme determina a Lei Complementar 07 - Plano Diretor de Natal, e dá outras providências. Disponível em: <http://www. estatutodacidade.com.br/>.

PMN (2003): Lei 2.050 de 06 de Janeiro de 2003. Define os empreendimentos e atividades que dependerão de elaboração de estudo prévio de impacto de vizinhança (EIV/RIV) e dispõe sobre sua elaboração e análise, nos termos do Estatuto da Cidade. Disponível em: <http://www.estatutodacidade.com.br/>.

PMPA (2004): Plano Diretor do Município de Porto Alegre. Prefeitura Municipal de Porto Alegre. Porto Alegre (RS), $77 \mathrm{pp}$.

PMRJ (2001): Projeto de Lei Complementar $N^{o}$ 25/2001: Dispõe sobre a Política Urbana do Município, instituindo o Plano Diretor da Cidade do Rio de Janeiro. Prefeitura da Cidade do Rio de Janeiro. Rio de Janeiro (RJ), 45 pp.

PMS (2004): Lei Municipal 7.482 de 21 de julho de 1998. Prefeitura Municipal de Sorrocaba. Disponível em <http://www.estatudodacidade.com.br>.

PMSP (2004): Plano Diretor do Município de São Paulo. Prefeitura da Cidade de São Paulo. Disponível em <http://portal.prefeitura.sp.gov.br/secretarias/planejamento/ plano_diretor/0004>.

PMT (1996): Lei Municipal 3.066 de 20 de dezembro de 1996. Prefeitura Municipal de Torres. Torres (RS), $22 \mathrm{pp}$.

PMV (2004): Plano Diretor do Município de Viçosa. Prefeitura Municipal de Viçosa. Disponível em $<$ http://www.estatudodacidade.com.br>.

RIO DE JANEIRO (1987a): Lei 1.130 de 12 de fevereiro de 1987. Diário Oficial do Estado do Rio de Janeiro, disponível em <http://www. lei.adv.br/>.

RIO DE JANEIRO (1987b): Decreto 9.760 de 11 de março de 1987. Diário Oficial do Estado do Rio de Janeiro, disponível em <http://www. lei.adv.br/>.

SANTA CATARINA (1982): Lei 6.063 de 24 de maio de 1982. Diário Oficial do Estado de Santa Catarina, disponível em <http://www. lei.adv.br/>.

SÃO PAULO (2003): Decreto 47.817 de 9 de maio de 2003. Diário Oficial do Estado de São Paulo, disponível em <http://www. lei.adv.br/>.

SÃO PAULO (2004): Resolução SH 17 de 26 de maio de 2004. Diário Oficial do Estado de São Paulo, disponível em <http://www. lei.adv.br/>.

SILVA, A.F.C. (2004): O solo urbano e os espaços de pobreza: os loteamentos na zona norte de natal. Disponível em <http://www.natal.rn.gov.br/sempla/paginas/noticias/solo_urbano.pdf>.

SOARES FILHO, J.G. (2001) Estatuto da Cidade: Lei 10.257. DP\&A Editora. Rio de Janeiro, $127 \mathrm{pp}$.

TUCCI, C.E.M. (2003): Drenagem Urbana. en Ciência e Cultura, vol 55. n. 4. Disponível em $<\mathrm{http}: / /$ cienciaecultura.bvs.br $>$. 


\section{Agradecimientos}

Los autores agradecen el Consejo Nacional de Desarrollo Científico y Tecnológico- CNPq por el apoyo al desarrollo del proyecto, por medio del proceso 150181/2004-3. 